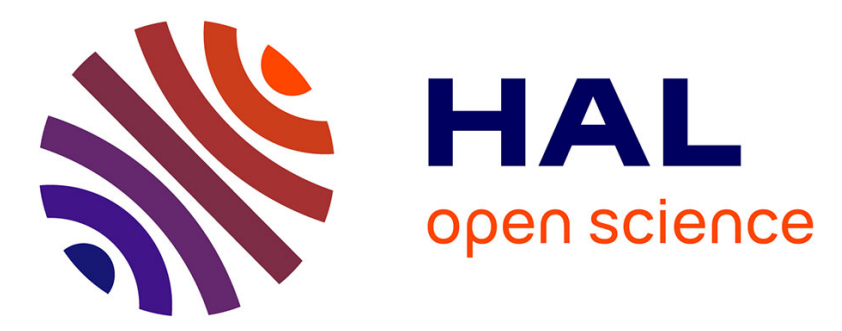

\title{
Dynamic energy-aware sensor configuration in multi-application monitoring systems
}

Ozgun Pinarer, Yann Gripay, Sylvie Servigne, Ozgovde Atay, Atilla Baskurt

\section{To cite this version:}

Ozgun Pinarer, Yann Gripay, Sylvie Servigne, Ozgovde Atay, Atilla Baskurt. Dynamic energy-aware sensor configuration in multi-application monitoring systems. Pervasive and Mobile Computing, 2017, 41, pp.192-204. 10.1016/j.pmcj.2017.08.005 . hal-01585263

\section{HAL Id: hal-01585263 https://hal.science/hal-01585263}

Submitted on 12 Sep 2017

HAL is a multi-disciplinary open access archive for the deposit and dissemination of scientific research documents, whether they are published or not. The documents may come from teaching and research institutions in France or abroad, or from public or private research centers.
L'archive ouverte pluridisciplinaire HAL, est destinée au dépôt et à la diffusion de documents scientifiques de niveau recherche, publiés ou non, émanant des établissements d'enseignement et de recherche français ou étrangers, des laboratoires publics ou privés. 


\title{
Dynamic Energy-Aware Sensor Configuration in Multi-Application Monitoring Systems
}

\author{
Ozgun Pinarer $^{\mathrm{a}, \mathrm{b}}$, Yann Gripay ${ }^{\mathrm{a}}$, Sylvie Servigne ${ }^{\mathrm{a}}$, Atay Ozgovde ${ }^{\mathrm{b}}$, Atilla \\ Baskurt $^{\mathrm{a}}$ \\ ${ }^{a}$ Univ Lyon, CNRS, France \\ INSA-Lyon, LIRIS, UMR5205, F-69621 \\ Email: name.surname@insa-lyon.fr \\ ${ }^{b}$ Galatasaray University \\ Ciragan Cad. No:36 34349 Istanbul, Turkey \\ Email: \{opinarer,aozgovde\}@gsu.edu.tr
}

\begin{abstract}
A typical pervasive monitoring system like a smart building depends on an infrastructure composed of hundreds of heterogeneous wireless sensor devices. Managing the energy consumption of these devices poses a challenging problem that affects the overall efficiency and usability. Existing approaches for sensor energy consumption typically assume a single monitoring application to consume sensor data and a static configuration for sensor devices. In this paper, we focus on a multi-application context with dynamic requirements and multi-modal sensor devices. We present 3SoSM, an approach to optimize interactions between application requirements and wireless sensor environment in real-time. It relies on an energy-aware dynamic configuration of sensor devices to lower energy consumption while fulfilling application requirements. To bind together sensor configuration and dynamic management of data streams, we design a sustainable multi-application monitoring system architecture for pervasive environments that collects application requirements for sensor data streams and optimizes them into sensor configurations. To demonstrate the effectiveness of our approach, a set of experiments are designed in the context of smart buildings. We comparatively evaluate our approach to show how dynamic sensor configuration for multiple monitoring applications indeed outperforms the mainstream duty-cycling method.
\end{abstract}

Keywords: pervasive environment, sensor data management, continuous query processing, wireless sensor network 


\section{Introduction}

Smart building applications have long gained attention in the scientific community and nowadays in the industry. Buildings, whether smart or not, are among the primary consumers of the available energy sources today. Therefore, considerable effort is poured into the design of smart buildings where energy consumption is tried to get decreased without sacrificing the satisfaction of the occupants. To accomplish this aim, the components of the building itself, as well as the context of its users, should be continuously monitored by means of streams of data from sensory inputs.

A typical pervasive monitoring system like a smart building consists of multi-modal wireless sensor devices that are equipped with sensing, processing and communication facilities. Sensing part can measure physical quantities about the environment with some given sampling rate (sampling frequency), processing part is able to do some computation on the measured values and communication part is able to listen and send data packets to other sensor devices. These deployed wireless devices are autonomous in terms of energy: they have limited energy and battery lifetime. Smart building technology mainly relies on such wireless sensing infrastructure. In this field, initial commercial solutions are Building Automation Systems where a specific set of functionalities such as heating and ventilation control and lighting management is implemented by a single vendor. However, as the sensor infrastructure is becoming a standard component incorporated into the design and construction of the modern buildings, flexible application development by a third party is becoming an issue rather than the infrastructure itself.

In this context, one of the main contributions of this paper is to allow multiple applications to exploit the same smart building infrastructure while considering energy consumption of the sensor devices in this infrastructure. Our energy-aware monitoring system continuously adapt to various application requirements, to building actual context and to user configuration. These applications operate on a network of multi-modal wireless sensor devices and use declarative continuous queries to exploit sensor data streams. Application requirements as declared by the application developer can be fulfilled in a multitude of ways: our method translates these requirements as energy efficient acquisition and transmission schedules for the wireless sensor 
network. We propose $\boldsymbol{S}$ mart-Service $\boldsymbol{S}$ tream-oriented $\boldsymbol{S}$ ensor $\boldsymbol{M}$ anagement (3SoSM), an approach to optimize interactions between application requirements and the wireless sensor environment in real-time. The core of our approach is the definition of a Schedule Time Pattern with acquisition, transmission and reception actions for each device configuration. The dynamic reconfiguration of devices is performed through the real-time update and optimization of Schedule Time Patterns according to application requirements that may change over time. With this approach, we expect to avoid unnecessary data measurements that may occur with static configuration and to promote grouped or even compressed data transmission when possible.

In this article, Section 2 presents an overview of our multi-application monitoring system architecture. Formalization of our approach is explained in Section 3 and the energy-aware optimization process is detailed in Section 4. Section 5 gives a brief description of our experimental platform to implement our approach and Section 6 describes the experiments we conducted. Experiment results are discussed in Section 7. Related works are given in Section 8 and finally, conclusions are given in Section 9.

\section{Overview of 3SoSM}

Our 3SoSM approach focuses on a multi-application monitoring system with multi-modal sensor devices (i.e. devices that can measure different physical quantities) and provides finer sensor configuration than duty-cycle and similar techniques. Our proposition of dynamic sensor management based on real-time application requirements is performed at the gateway level, in order to optimize energy consumption of sensor devices independently from the application layer and/or the query engine.

\subsection{Monitoring Architecture for Smart Building Applications}

In this study, we adopt a "declarative monitoring architecture", built upon a Pervasive Environment Management System (PEMS) as presented in $[1,2,3]$. Using declarative (SQL-like) continuous queries, an application can easily interact with distributed devices like sensors. Figure 1 presents our declarative monitoring application that has 4 main layers: Application, PEMS Query Engine, PEMS Gateway and WSN (Wireless Sensor Network). Application layer provides end-user access to the monitoring system. Application requirements are defined at this layer and are declaratively expressed as a set of continuous queries over distributed services [1]. PEMS 
Query Engine is responsible for managing query executions of queries coming from the Application layer. This layer integrates sensor devices as nonconventional, dynamic and heterogeneous data sources. To manage that, it includes a continuous query engine that interacts with services provided by the environment, in particular sensor services. PEMS Gateway stands for managing bidirectional communication and interactions between PEMS Query Engine and WSN. WSN layer represents wireless sensor devices that acquire physical quantity measures and can communicate with other sensor devices and physical gateways.

We use the Service-Oriented Continuous Query (SoCQ) framework [1] for the PEMS Query Engine. The SoCQ engine handles a multi-application mechanism and supports multiple parameterized stream subscriptions to the same device. Moreover, it supports real-time user configuration of applications and context-aware applications through queries that can dynamically com-

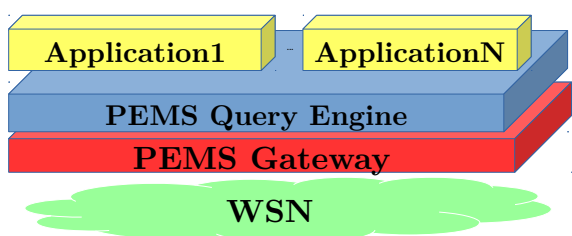

Figure 1: Declarative Monitoring Architecture. bine data, streams and services. The user may insert complex continuous queries with conditions over data streams to trigger new interactions with services during their execution. Interactions with services (discovery, invocations of methods, subscriptions to data streams) are handled by PEMS Gateway. However, the SoCQ Engine itself does not overcome the reconfiguration of sensor devices, and thus assumes a pre-existing static configuration. Like for other approaches presented in the Related Works section, it is an issue for the energy enhancement of the system.

\subsection{From Dynamic Application Requirements to Dynamic Sensor Configu- rations}

We consider that applications define their requirements as a set of continuous queries over parameterized data streams produced by sensor devices. For instance: "Application A1 computes the average temperature over the last 10 minutes, with an update every 5 minutes, with an accuracy of 1 second and a maximum latency of 1 minute". In this example, the application requests temperature measure data streams from all devices with temperature sensors. To express the application requirements concerning sensor data management, we consider the following parameters targeting sensor measures: • temporal 
window introduces the time interval for calculating the result (for the given example: $10 \mathrm{~min}$ ); • update periodicity stands for the refreshing rate of the result (5 min); • acquisition periodicity represents the temporal accuracy of measures (1 sec); • maximum latency presents the maximum acceptable delay between the acquisition of data and its transmission to the PEMS layer for result calculation (1 min). temporal window and update periodicity concern the computing of the result, whereas acquisition periodicity and maximum latency are related to acquisition/transmission of data by sensor devices.

Here, we propose a novel approach: management and optimization of the real-time application requirements to enhance the energy consumption of the whole system. To fulfill the application requirements, we define a Schedule Time Pattern for each sensor device and configure devices accordingly. Our approach assumes that sensor devices are dynamically configurable [4].

\section{3SoSM Formalization}

We consider that application requirements are parameterized stream subscription requests and the network topology is a tree topology. A solution to our optimization problem is individual Schedule Time Patterns, namely SCO-PATTERNS.

\subsection{Application Requirements}

Let $D$ be the set of wireless sensor devices in the WSN environment. Each sensor device $d_{i} \in D$ may have multiple modalities to acquire different physical quantity measures $m_{i} \in M$, e.g., temperature, humidity. Application requirements are defined in terms of data source requirements: targeted sensors $d$ and requested measures $m$; and of temporal requirements: temporal window $\beta$, update periodicity $p^{u p d}$, acquisition periodicity $p^{a c q}$ and maximum latency $\lambda$. The unit is the second (or millisecond, if required).

For clarity, we do not present $\beta, p^{\text {upd }}$ in this paper. We then represent application requirements on sensors at a given time instant by a set of parameterized subscription requests $S=\left\{s_{1}, s_{2}, \ldots, s_{n}\right\}$, where:

$s_{i}=\left(d_{i}, m_{i}, p_{i}^{a c q}, \lambda_{i}\right) \in D \times M \times \mathbb{N}^{+} \times \mathbb{N}^{+}$

Example 1. Suppose two applications with the following requirements on 6 sensors:

Application 1: Temperature $\left(m_{T}\right)$ of sensors $d_{1}, d_{3}, d_{5}$ with an acquisition 
periodicity of 2 sec and a latency of $4 \mathrm{sec}$;

Application 2: Humidity $\left(m_{H}\right)$ of sensors $d_{3}, d_{4}, d_{5}, d_{6}$ with an acquisition periodicity of $5 \mathrm{sec}$ and a latency of $3 \mathrm{sec}$.

Based on the previous notation, all application requirements are represented by a set of parameterized subscription requests: $S=\left\{\left(d_{1}, m_{T}, 2,4\right),\left(d_{3}, m_{T}, 2,4\right),\left(d_{5}, m_{T}, 2,4\right),\left(d_{3}, m_{H}, 5,3\right),\left(d_{4}, m_{H}, 5,3\right)\right.$, $\left.\left(d_{5}, m_{H}, 5,3\right),\left(d_{6}, m_{H}, 5,3\right)\right\}$

\subsection{Network Topology}

To transmit data to the central base station (where results are computed), we suppose that deployed wireless sensor devices construct a logical tree (tree topology) where each sensor has only one neighbor to deliver data packets. This tree structure provides a uni-path for each sensor to reach the base station (sink device). In fact, our optimization algorithm relies on known and unique paths, as it does not consider a choice of network paths between two devices. Integrating more complex network topologies, such as mesh networks, would be a future work. However, the tree topology may be updated each time application requirements change, as it triggers a new optimization. For instance [5] proposes a new technique to organize the sensor devices that is likely preferable for outdoor monitoring systems: P-SEP (a prolong stable election routing algorithm). The authors consider two-level sensor device heterogeneities: advanced and normal devices. They propose a clustering mechanism and present new cluster head selecting policy to extend the lifetime of the system (lifetime of the system is evaluated in terms of FND (First Node Dies)). P-SEP puts forth efficient simulation results and network lifetime. Although we do not tackle routing issues, applying our dynamic network behavior due to the dynamicity of the application requirements, with the P-SEP mechanism could produce concrete improvements on the lifetime of the system. In our case, with our assumptions about the network, each sensor device has its own role in the topology based on the current applications and its location: Source (responsible for the data acquisition and transmission) and/or Relay (responsible for the reception and re-transmission), or Sink (responsible for only the reception).

Example 2. A tree topology with 6 multi-modal sensor devices and a base station is illustrated in Figure 2. A tree topology has a layered form based on the distance to the base station. 


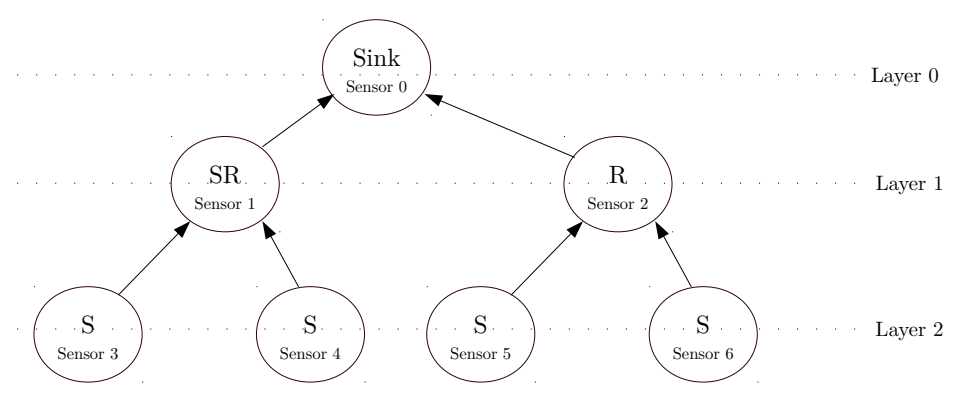

Figure 2: A tree-structured topology and different sensor device roles: Source (S), Relay (R), Source+Relay (SR), Sink.

\subsection{SCO-PATTERN}

The idea behind the 3SoSM approach is to create a Schedule Time Pattern for each sensor device. This pattern, described in [6] is a schedule composed of sensor actions, here with three types of actions: acquisition A, reception $R$, transmission T. Based on their roles, only certain actions are pertinent to sensor devices. Sensor devices can configure themselves with a Schedule Time Pattern, and then execute periodically the scheduled actions: acquire a measure and store it in a memory buffer, transmit all buffered measures one hop towards the sink (and empty the buffer), receive measures from a lower-layer device and store it in a memory buffer.

We call this pattern sensor configuration oriented pattern or SCOPATTERN. This pattern consists of timestamped events $(<$ timestamp, action $>$ couples) and a length $\ell$ (or periodicity) of that pattern. These actions are enclosed by the length of the pattern: event timestamps are in time interval $[0 ; \ell[$. A SCO-PATTERN is denoted by: $\mathrm{P}=\left(\left\{\left(t_{i}, a_{i}\right)\right\}, \ell\right)$ with $\ell \in \mathbb{N}^{+}, t_{i} \in\left[0 ; \ell\left[, a_{i} \subset\left\{A_{m}, T, R\right\}\right.\right.$ where $A_{m}$ indicates the data acquisition of the physical measure $m$ such as $A_{T}$ for temperature, $A_{H}$ for humidity, etc.

\section{3SoSM: Energy-Aware Optimization Process}

To optimize the energy consumption of the devices, since the communication costs are the most significant part of the energy budget [7], we want to minimize the number of transmission and reception actions of each device: less communication means less consumed energy. We first construct "preliminary" SCO-PATTERNs that have a common length with only acquisition actions (Acq) for all the devices. It is then necessary to insert the transmission $(\mathrm{Tx})$ and reception $(\mathrm{Rx})$ actions in these SCO-PATTERNs to obtain a solution, composed of the set of complete SCO-PATTERNs. A solution is 
valid if all acquired data traverse the network topology from their source device to the base station before the "latency" associated with that data expires. The optimal solution is a valid solution that minimizes the number of Transmission / Reception actions of each device.

The objective of the optimization process is to transform the application requirements into a global schedule and then to build individual SCOPATTERns to configure each device. Application requirements are represented by a set of subscription requests $S=\left\{s_{1}, s_{2}, s_{3}, \ldots\right\}$. Hence the energy-aware optimization process can be expressed as a transformation function that gets a set of subscription requests (all subscription requests from all applications) and a network topology $\mathcal{N}$ and produces a SCO-PATTERN for each device: $f\left(\left\{s_{1}, s_{2}, \ldots, s_{n}\right\}, \mathcal{N}\right)=\left\{\mathrm{P}_{d_{1}}, \mathrm{P}_{d_{2}}, \ldots, \mathrm{P}_{d_{m}}\right\}$.

\subsection{Preliminary SCO-PATTERN}

The preliminary SCO-PATTERN is specific for each sensor device. It is periodic, and its length indicates the periodicity. Each sensor device may have different acquisition periodicities from different applications. However, in order to build a global view of the network, a common length is required: it is the lowest common multiple of acquisition periodicities from all subscription requests. Moreover, the length of the schedule should be greater than any latency of the subscription requests to properly handle data expiration time: The final length is the lowest multiple of this initial common length greater than any data expiration time.

Example 3. The common length of the patterns (for the given applications in Example 1) is calculated as:

Initial common length $\ell_{\min }=\operatorname{LCM}(2,2,2,5,5,5,5)=10$

Maximum latency $\lambda \max =\operatorname{MAX}(4,4,4,3,3,3,3)=4$

Final length $\ell_{\text {schedule }}=10 \mathrm{sec}>\lambda_{\max }$

Then, based on the periodicity of subscription requests, acquisition actions are defined. Furthermore, each acquisition action is tagged with its maximum latency (from the subscription request).

Example 4. Preliminary SCO-PATTERN for $d_{3}$ with subscriptions $\left\{\left(d_{3}, m_{T}, 2,4\right),\left(d_{3}, m_{H}, 5,3\right)\right\}$ :

$P=\left(\left\{\left(0,\left\{A_{T}^{4}, A_{H}^{3}\right\}\right),\left(2, A_{T}^{4}\right),\left(4, A_{T}^{4}\right),\left(5, A_{H}^{3}\right),\left(6, A_{T}^{4}\right),\left(8, A_{T}^{4}\right)\right\}, 10\right)$ 


\subsection{Transmission Constraint and Optimization Goal}

To form a global schedule, we adopt a similar approach to Galpin et al. [8]. A predefined granularity determines the slots in the time space: the schedule is divided into time-slots and each slot may be filled with sensor actions. For the following examples, granularity is set to $0.5 \mathrm{sec}$ (e.g. 20 slots for 10 seconds).

The major constraint of the system is that all the acquired data must pass through the network topology up to the base station before the expiration of their "latency" in order to fullfill the application requirements. A transmission action on a sensor device during a single time slot stands for a transmission of all the data (acquired by itself and received from lower-layer neighbors) not yet sent, including acquisitions on the same time slot. This transmission action requires a reception action at the same time slot on the sensor device that receives the data. Besides there are other constraints due to the radio protocol such as a device can only receive data from a single device at a time on the same time slot and a device can not receive and send on the same time slot.

\subsection{Searching Optimal Communication Slots}

We propose an optimization algorithm to choose the optimal communication slots from the preliminary SCO-PATTERN. The optimization process starts by propagating transmission and reception constraints due to the acquisition events in a bottom-up process, and then analyzes the possible reception actions of the base station and tries to find the most energy efficient communication slots, and finally propagates those choices to lower layers and continues the analyze in a top-down process.

\subsubsection{Schedule Table Initialization}

We use a schedule table to represent the sensor action slots for $\mathrm{Tx} / \mathrm{Rx}$. Each sensor device has its own schedule and each schedule is composed of two parts: transmission and reception part. The length of the schedule is defined by the common length of preliminary SCO-PATTERNs. Each line of the schedule table corresponds to an acquired measure: its transmission action for the transmission part and its reception action for the reception part.

The transmission part indicates the possible transmission slots for the sensor device for each acquired measure. According to the latency information $\lambda$, every sensor device has the initiative to keep the data (instead of 
sending it immediately) and send it at an optimal later moment under the condition that this acquired data arrives at the base station before the expiration of its latency. The reception part represents the possible reception slots for each data coming from lower-layer neighbors (zero, one or several depending on the network topology). Schedule Table for Sensor 3, Sensor 1 and Sensor 0 (sink device) are illustrated in Figure 3.

We propagate bottom-up constraints for data transmission and reception based on latencies (from preliminary SCO-PATTERNs) and on the network topology.

Transmission Part. Information from the preliminary SCO-PATTERN is used to fill the transmission part. For a given sensor device, each acquired data and each received data is represented as a line in the transmission part. A line is filled with the residual latency information starting from the time slot where the device acquires the data or from the time slot following the time slot where the device received the data, and until residual latency reaches 0 (in fact, 1 time slot before at 1 hop from the sink, 2 time slots at 2 hops, etc.).

For each device $d, T_{d}=\left[e_{i, j}\right]$ is a $2 \mathrm{D}$ matrix with dimensions $m_{d} \mathrm{x} n . m_{d}$ is the number of data to transmit and $n$ stands for the number of time slots. $e_{i, j}$ represents a potential transmission event of data $i$ at time slot $j: e_{i, j}=\lambda_{i, j}$ is the residual latency, $e_{i, j}=0$ means that data $i$ can not be transmitted at that time slot.

Example 5. Transmission parts of the schedules of Sensors 3 and 1 are presented respectively in Figure 3a, 3b. For instance, Sensor 3 measures temperature and humidity values. For A2\#1 (first acquisition for humidity), the latency is 3sec. Hence, to send that data, Sensor 3 has 5 slots ( $t=0,0.5,1$, $1.5,2 \mathrm{sec})$ before the latency is expired as it is 2 hops from sink. Transmission part of the schedule of Sensor 1 covers transmission of its own data and also received data from Sensor 3 and 4 (see Fig 3b).

Reception Part. For a given sensor device, the reception part is based on the transmission part of the schedules of lower-layer neighbors. Each line represents a data to receive and is linked to one line from the transmission part of the source device. Possible reception slots are filled with the data residual latency from the corresponding transmission time slot.

For each device $d, R_{d}=\left[e_{i, j}\right]$ is a $2 \mathrm{D}$ matrix with dimensions $m_{d} \mathrm{x} n$ where $m_{d}$ is the number of data to receive and $n$ stands for the number of time slots. 


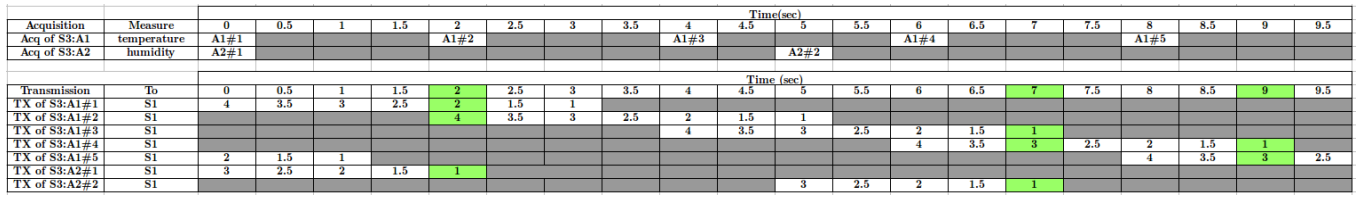

(a) Schedule of Sensor 3 (Acquisition + Transmission).

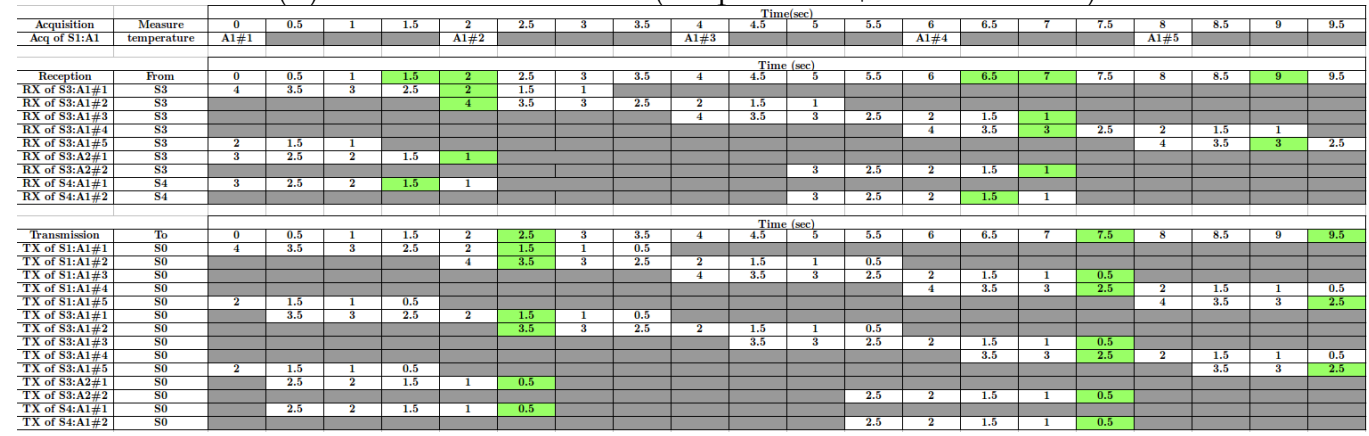

(b) Schedule of Sensor 1 (Acquisition + Reception + Transmission).

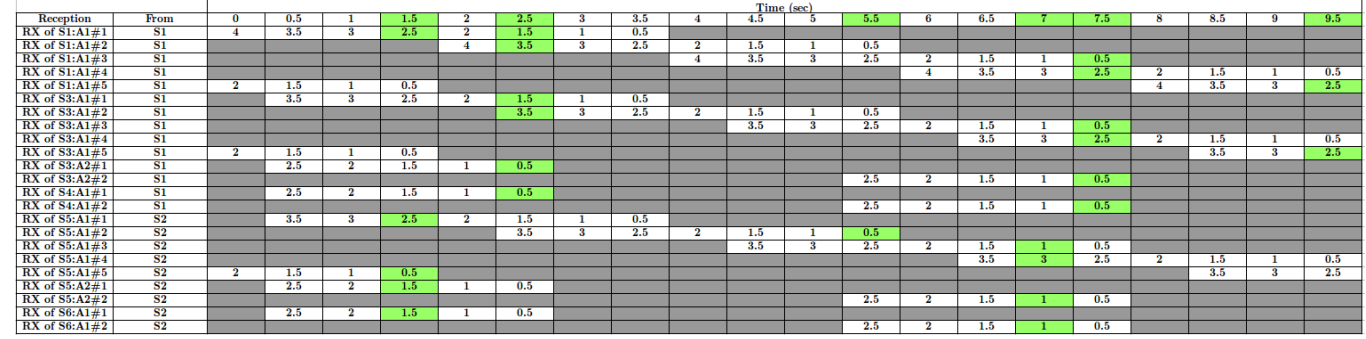

(c) Schedule of Sensor 0 (Sink device) (Reception).

Figure 3: Schedules (highlighted slots are the optimal communication slots based on our process).

$e_{i, j}$ represents a potential reception event of data $i$ at time slot $j: e_{i, j}=\lambda_{i, j}$ is the residual latency, $e_{i, j}=0$ means that data $i$ can not be received at that time slot.

Example 6. For the given example, reception parts of the schedule for Sensor 1 and Sink are represented respectively in Figure $3 b$ and 3c. For instance, reception part of Sensor 1 represents the possible reception slots for each acquired data of Sensor 3 and 4 with their residual latency.

\subsubsection{Optimal Choice of $T x / R x$ Slots}

For each device $d_{i}$, potential transmission and reception slots are now identified in their schedule table (matrices $T_{d_{i}}$ and $R_{d_{i}}$ ). The optimization 
process should now choose the most energy-efficient slots, in order to minimize the number of $\mathrm{Tx} / \mathrm{Rx}$ slots for each device. From the reception part of the schedule, the algorithm tries to group the receptions from the same sensor device. For each time slot, it calculates the total number of possible receptions for each neighbor. The algorithm searches the latest slot in which maximum number of reception can occur. The latest slot indeed creates more opportunities for the lower-layer devices. Otherwise, it would force the lower-layer devices to send the data earlier.

In our example, the algorithm analyzes reception part of Sensor 0 (Figure 3c). It starts by deciding the best communication slot for Sensor 1 and then for Sensor 2. For Sensor 1, the algorithm chooses $t=7.5$ since the maximum of possible receptions is 6 and the latest occurrence of 6 is at $t=7.5$. Slot occupancy is updated: the slot for $t=7.5$ becomes occupied. Then the algorithm repeats the same decision process for Sensor 2 and chooses $t=7$, as the latest possible occurrence of value 4 is at $t=7$. Although $t=7.5$ is the actual latest maximum, this slot is already occupied, so the communication slot for Sensor 2 is set to $t=7$. The status of the process after the first iteration is given in Figure 4. The algorithm updates the reception part of the device by defining the unique $\mathrm{Rx}$ slot for each concerned data, then it repeats the same process to decide communication slots for remaining data. This iteration continues until all data have a defined Rx slot.

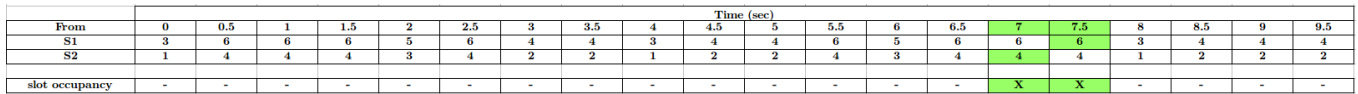

Figure 4: Searching for Optimal Rx Slot on Sensor 0 Slot occupancy: Free -, Occupied X. devices. This top-down process continues until all $\mathrm{Tx} / \mathrm{Rx}$ slots are defined for all devices. The finalized reception part of Sensor 0 is illustrated in Figure 3c. The green slots are the optimal slots found by our algorithm. Finalized schedules of Sensor 1 and Sensor 3 are also presented in Figure 3. 


\subsection{Building Complete SCO-PATTERN}

Once schedules are finalized after searching optimal communication slots for each device, the last step is to extract $\mathrm{Tx} / \mathrm{Rx}$ events to obtain the complete SCO-PATTERNS.

Example 7. Here are the SCO-PATTERNs for the sensor devices of our example:

$\mathrm{P}_{1}=\left(\left\{\left(0,\left\{A_{T}\right\}\right),(1.5,\{R\}),\left(2,\left\{A_{T}, R\right\}\right),(2.5,\{T\}),\left(4,\left\{A_{T}\right\}\right),\left(6,\left\{A_{T}\right\}\right),(6.5,\{R\}),(7,\{R\}),(7.5,\{T\})\right.\right.$,

$\left.\left.\left(8,\left\{A_{T}\right\}\right),(9,\{R\}),(9.5,\{T\})\right\}, 10\right)$

$\mathrm{P}_{2}=(\{(0.5,\{R\}),(1,\{R\}),(1.5,\{T\}),(5,\{R\}),(5.5,\{T\}),(6,\{R\}),(6.5,\{R\}),(7,\{T\})\}, 10)$

$\mathrm{P}_{3}=\left(\left\{\left(0,\left\{A_{T}, A_{H}\right\}\right),\left(2,\left\{A_{T}, T\right\}\right),\left(4,\left\{A_{T}\right\}\right),\left(5,\left\{A_{H}\right\}\right),\left(6,\left\{A_{T}\right\}\right),(7,\{T\}),\left(8,\left\{A_{T}\right\}\right),(9,\{T\})\right\}, 10\right)$

$\mathrm{P}_{4}=\left(\left\{\left(0,\left\{A_{H}\right\}\right),(1.5,\{T\}),\left(5,\left\{A_{H}\right\}\right),(6.5,\{T\})\right\}, 10\right)$

$\mathrm{P}_{5}=\left(\left\{\left(0,\left\{A_{T}, A_{H}\right\}\right),(1,\{T\}),\left(2,\left\{A_{T}\right\}\right),\left(4,\left\{A_{T}\right\}\right),\left(5,\left\{A_{H}, T\right\}\right),\left(6,\left\{A_{T}, T\right\}\right),\left(8,\left\{A_{T}\right\}\right)\right\}, 10\right)$

$\mathrm{P}_{6}=\left(\left\{\left(0,\left\{A_{H}\right\}\right),(0.5,\{T\}),\left(5,\left\{A_{H}\right\}\right),(6.5,\{T\})\right\}, 10\right)$

Once SCO-PATTERns are completed, they are sent by the Gateway to sensor devices to configure them. Sensor devices then execute the given actions periodically, and data flow to the base station where continuous queries are computed. If an application changes its requirements, the configuration process starts again from the beginning.

\section{Experimental Platform}

In this section, we present the tools used in this study: SoCQ Engine as a continuous query engine and WSNet simulator for the wireless sensor network environment.

\subsection{Continuous Query Engine: SoCQ Engine}

As a framework for PEMS, we use the SoCQ (Service-oriented Continuous Query) framework [1]. It takes a data-oriented perspective on the pervasive environment: it provides a unified view and access to the various and heterogeneous resources available in the environment. Pervasive applications can then be created in a declarative way using service-oriented continuous queries over such an environment.

Within the SoCQ framework, XD-relations (eXtended Dynamic Relations) represent standard relations, that may be updated, or data streams, that continuously produce data. The definition of XD-relations can also include virtual attributes and binding patterns that together enable queries to interact with distributed services: service discovery, method invocation, stream 
subscription. Queries may be one-shot queries (like standard SQL queries) or continuous queries (with a dynamic result, like a stream). Furthermore, invocations of service methods and subscriptions to service streams can be parameterized.

We illustrate SoCQ in the context of Smart Building. Table 1 shows a discovery query, the resulting XD-Relation, then a one-shot query and a continuous query over this XD-Relation. The discovery query (DISCOVER Services) searches for sensor services that provide a location, a method to get the current temperature, and a continuous stream of temperature measures. The resulting XD-Relation TemperatureServices has one ServiceID attribute (here, the URI of a sensor), a Location, and a virtual attribute for Temperature. When executed, the one-shot query (SELECT ONCE) selects services located in a given room and retrieve the current temperature by invoking getTemperature method. While executing, the continuous query (SELECT STREAMING) subscribes to the temperature stream of every discovered service to build a resulting data stream. If new services are discovered and/or some services become unavailable, the continuous query automatically adapts the corresponding stream subscriptions.

Table 1: Example of SoCQ queries for a Smart Building.

\begin{tabular}{|l|l|l|}
\hline CREATE RELATION TemperatureServices ( & & \\
ServiceID SERVICE, & & \\
Location STRING, & SELECT * & SELECT * \\
Temperature NUMBER VIRTUAL & ONCE FROM TemperatureService & STREAMING UPON insertion \\
) USING ( & WHERE Location = "501.340" & FROM TemperatureServices \\
getTemperature[ServiceId]():(Temperature), & USING temperature[1]; \\
temperature[ServiceId]():(Temperature) STREAMING & USING getTemperature; & \\
AS DISCOVER SERVICES PROVIDING & & \\
PROPERTY Location STRING, & & \\
METHOD getTemperature ( ) : ( NUMBER ), & & \\
STREAM temperature ( ): ( NUMBER ) & & \\
\hline
\end{tabular}

\subsection{WSN Simulator: Modified WSNet}

In our platform, we integrated the WSN simulator WSNet [10]. WSNet is a modular event-driven simulator, more precisely a discrete event simulator (DES). WSNet adopts basic functionality of $D E S$ : in order to avoid simulating every time splice, the time line is split into events and no change is presumed to occur in the system between consecutive events; thus the simulation can directly jump in time from one event to the next. However, as the PEMS works on real-time, we modified the time scheduler of the WSNet simulator to avoid time scheduling difference: we introduced a time factor to run experiments in real-time or $n$-times faster $(\times 10, \times 20 \ldots)$. 


\subsection{Gateway: 3SoSM Gateway}

In a declarative monitoring architecture (see Figure 1), the Gateway is a technical bridge between two environments: it manages interactions and bidirectional communication between the PEMS Query Engine and the WSN. We implemented the 3SoSM principles in a 3SoSM Gateway. It is implemented in Java, and interacts with the SoCQ engine and WSNet. 3SoSM Gateway has two primary modules: the Service Manager, that manages SoCQ services representing available sensor devices (real devices or simulated devices); and the Subscription Manager, that continuously analyses application requirements to generate new SCO-PATTERNs for sensor devices when required. In a typical scenario, multiple applications launch continuous queries concerning sensors to the PEMS. The gateway manages required parameterized stream requests and, according to the 3SoSM optimization process, generates optimal sensor configurations. With our integrated prototype, we can execute multiple SoCQ queries that dynamically subscribe to streams provided by WSNet sensor devices. The Gateway transparently optimizes the subscriptions and configures the WSNet sensor devices with SCO-PATTERNs.

\section{Experiments}

\subsection{Experimental Setup}

The simulations are performed using the modified WSNet. We simulate one part of the topology of our physical platform SoCQ4Home deployed in our LIRIS laboratory: 70 sensor devices are located at specific positions over a floor of the building $(10 \mathrm{~m} \times 60 \mathrm{~m} \times 4 \mathrm{~m})$. The topology is illustrated in Figure 5. The deployed sensor devices have fixed positions during the simulation and we consider that they have enough energy until the end of the simulation. During the simulation process, the initial energy level of sensor devices is set on purpose to emphasize the lifetime difference between both cases.

We adopt known pervasive environment communication protocol Zigbee IEEE 802.15.4, simulated with a UDG propagation model (which is a strong simplification for building environments, as it does not take into account environmental effects), 35m transmission range, and basic radio module states for devices (idle, active, sleep, transmission, reception). Calculation of energy consumption is based on CPU and radio components, adopted from [7]: $\mathcal{E}_{\text {device }}=\mathcal{E}_{C P U}+\mathcal{E}_{\text {radio }}=\mathcal{E}_{C P U}+\left(\mathcal{E}_{\text {sleep }}+\mathcal{E}_{T X}+\mathcal{E}_{R X}\right)$, with $\mathcal{E}_{\text {component } / \text { mode }}=$ 


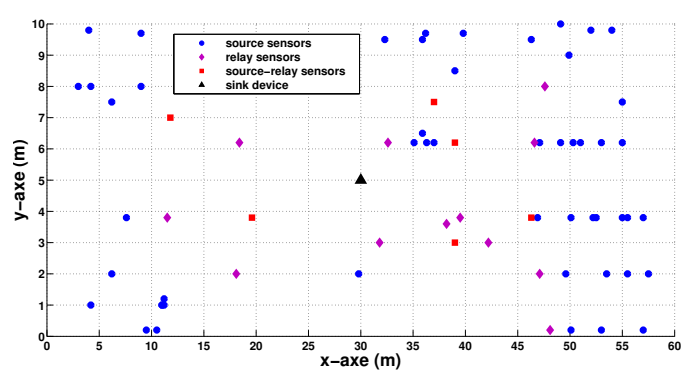

Figure 5: Topology of the network

(Sensor roles are set based on the Application 1 of the scenario given below).

$I_{\text {mode }} . V_{\text {drain }} . \Delta t_{\text {mode }}$. This model is a well-known energy consumption calculation model preferred by many researchers. For instance the paper [11] proposes a temperature-aware task mapping approach for the mapping of multiapplication to NoC-based many-core systems by balancing workloads among cores. In our study, we adopt a similar approach to calculate the energy consumption of all devices. We implemented sensor devices for WSNet so that they can reconfigure themselves when they receive a new SCO-PATTERN packet, and so that WSNet can monitor their simulated energy consumption while executing sensor actions.

\subsection{Experimental Scenario}

To evaluate our approach, we compare 2 types of architecture:

1) an architecture with basic duty-cycle WSN devices, requiring a static configuration predefined according to scenario requirements;

2) an architecture with 3SoSM approach where WSN devices can be dynamically configured with SCO-PATTERNs generated by the 3SoSM Gateway.

We design four scenarios: "Temperature of Occupied Rooms", "Comfort Temperature Range", "Room occupancy based on $\mathrm{CO}_{2}$ emission" and "Luminosity of rooms". Each scenario is performed during one day (1440min). Here, we present only one of the performed scenarios due to space limitations.

Scenario "Temperature of Occupied Rooms": One of the features of a typical smart building is monitoring temperatures and occupancy of the environment. Here we suppose that occupancy sensor devices are not always active but has their own duty cycle for detecting the motion.

In this scenario, there exists two applications:

Application 1: Monitor the temperature of each room with a data acquisition every $15 \mathrm{sec}$ and a latency of $60 \mathrm{sec}$. 
Application 2: Monitor temperature of occupied rooms with a data acquisition every $1 \mathrm{sec}$ and a latency of $4 \mathrm{sec}$.

Here, Application 1 requests the temperature from all temperature sensor devices with given application requirements. Application 2 also requests a temperature with a special constraint: it intends to track the temperature only for occupied rooms. For this application requirement, occupancy and temperature sensor devices are needed.

For the duty-cycle approach, we adopt a static configuration. In this case, we consider the worst case situation: occupation may be true at any location during the simulation. Based on this constraint, sensor devices are configured with: $p^{a c q}=1 s e c, p^{t x}=4 \sec$ (periodic transmission)

In our approach, we propose a dynamic sensor configuration based on the real-time context. To respond to Application 1 and Application 2, sets of SoCQ queries are implemented. For Application 2, from the occupancy stream, we extract the location attribute of the occupied rooms. Then, we list the temperature sensor devices that are located in these rooms and implement a new stream query to subscribe to those temperature services with given application requirements. Since these are continuous queries, once a room is not occupied any more or an unoccupied room become occupied, lists are refreshed and new subscriptions or unsubscriptions are handled in realtime. Then, the 3SoSM Gateway creates schedules for each sensor device and generates SCO-PATTERNs to configure sensor devices accordingly.

Briefly, Application 1 receives temperature data from relevant devices under every condition. However, Application 2 has a remarkable condition: at least one room should be occupied so that it starts to receive temperature data. Here, there are two subscriptions to the same set of temperature sensor devices located in occupied rooms with different application requirements. During the simulation (one day), energy consumption (in terms of joules) of every sensor are monitored periodically and logged.

Besides, three more scenarios are performed. Scenario 2 consists of tracking temperature of each room and requesting more frequently temperatures for rooms that are out of the current comfort temperature range. Scenario 3 is based on the occupancy information retrieved from $\mathrm{CO}_{2}$ emission detected in the room and scenario 4 is related to the luminosity of a classroom. For these scenarios, two applications with different application requirements are executed during a day. 


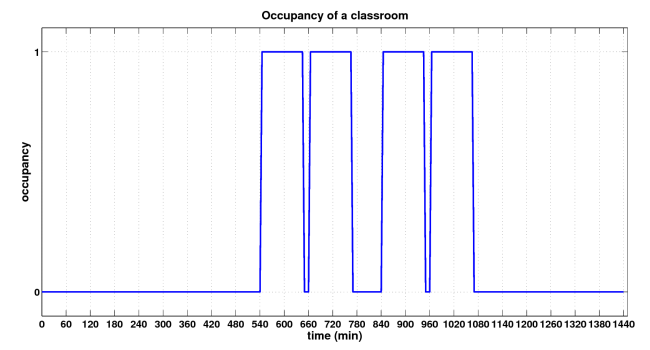

(a) Occupancy of a room $(24 \mathrm{~h})$.

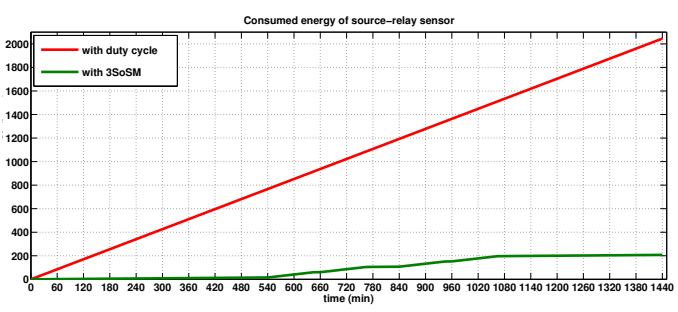

(b) Average consumed energy (in joule) of a source-relay device $(24 \mathrm{~h})$. Upper line for duty cycling, lower line for 3SoSM.

Figure 6: Occupancy information and Energy consumption of the most significant device type: source-relay device with duty cycling and with our approach 3SoSM.

\section{Results and Discussion}

During the experiments, energy consumption of each sensor device is monitored. Figure 6 shows the average energy consumption of source-relay devices. As a remark, the sink device is not considered as a part of this energy consumption calculation. Here, we present the results of the first scenario in detail for one day (1440min). Firstly, the room is not occupied (see Figure 6a), hence the 3SoSM Gateway creates a schedule and configures the relevant sensor devices according to that situation. Once the room is detected as occupied, the 3SoSM Gateway re-creates a schedule and re-configures the relevant sensor devices. In fact, the 3SoSM Gateway creates a new schedule and configures sensor devices to adapt to each contextual change in the environment. Thus, adapting the system to the dynamic context avoids sensor devices from unnecessary data acquisitions and transmissions. Hence, the economy of energy can be achieved with this context-awareness, as shown in Figure 6b.

Evolution of the energy consumption for the WSN can be observed with heat maps as well. Figure 7 represents the heat map of the network based on the energy level of the sensor devices at the end of the simulation $(t=24 \mathrm{~h})$, with duty-cycle method (Figure 7a) and with our 3SoSM approach (Figure $7 \mathrm{~b}$ ), according to the given topology (see Figure 5). With the settings of the scenario "Temperature of Occupied Rooms", we observe that our approach 3SoSM enhances the lifetime of the sensor devices significantly. For source-relay sensor devices, with given application requirements, 3SoSM extends their lifetime by 8.9 times on average. 


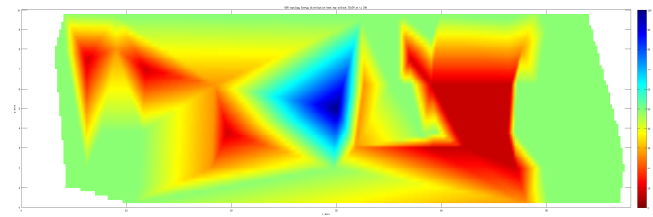

(a) Heatmap of remaining energy at $t=24 \mathrm{~h}$ with duty cycle.

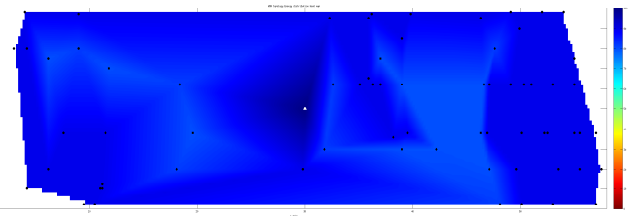

(b) Heatmap of remaining energy at $\mathrm{t}=24 \mathrm{~h}$ with $3 \mathrm{SoSM}$.

Figure 7: Heatmap of remaining energy with duty-cycle and with 3SoSM.

Table 2: Summary of the Experiments.

\begin{tabular}{|c|c|c|c|c|c|c|}
\hline \multirow[b]{2}{*}{ Scenarios } & \multicolumn{4}{|c|}{ Application Parameters } & \multicolumn{2}{|c|}{ Lifetime (day) } \\
\hline & $\begin{array}{l}\text { Appli } \\
p^{a c q} \\
(\sec )\end{array}$ & $\begin{array}{c}1 \\
\lambda \\
(\mathrm{sec})\end{array}$ & $\begin{array}{l}\text { Appl } \\
p^{a c q} \\
\text { (sec) }\end{array}$ & $\begin{array}{c}2 \\
\lambda \\
(\mathrm{sec})\end{array}$ & Duty cycling & 3SoSM Approach \\
\hline $\begin{array}{c}\text { Temperature of } \\
\text { Occupied Rooms }\end{array}$ & 15 & 60 & 1 & 4 & 15 & 146 \\
\hline $\begin{array}{c}\text { Comfort } \\
\text { Temperature Range } \\
\end{array}$ & 20 & 60 & 10 & 20 & 9 & 90 \\
\hline $\begin{array}{l}\text { Room Occupancy } \\
\text { based on } \mathrm{CO} 2\end{array}$ & 30 & 120 & 15 & 60 & 11 & 95 \\
\hline Luminosity of Rooms & 30 & 100 & 20 & 80 & 10 & 34 \\
\hline
\end{tabular}

By applying our approach to given application requirements, we optimize the energy consumption and reduce the unnecessary communication cost. Obtained results on energy saving and lifetime extension depend on the application requirements and the context of the application such as temperature and presence. For the current settings, the most unfavorable case is the situation where every room is occupied during the entire simulation time. Even in that case, 3SoSM achieves a concrete energy enhancement since the regular duty-cycle approach does not benefit from latency requirement which provides a tangible energy saving by allowing grouped transmission of multiple acquired data.

Besides, the details of the other performed scenarios are summarized in Table 2 for each scenario, application requirements are given and the average lifetime of source-relay sensor devices are presented. We also obtain a significant energy enhancement and a lifetime extension by avoiding unnecessary communication.

However, balancing the workload of the network over the sensor devices like [11] introduces, advanced clustering mechanism like [5] presents, may further enhance the network lifetime and the obtained results. [5] presents that with a clustering mechanism and selecting cluster headers in an optimal way may extend the lifetime of the network better than any other cluster 
based networks. With more populated topologies and with massive subscription requests, a discussion about the optimization process appears: in which conditions our approach may fail to find the optimal slots on the schedules (since a slot is reserved for a single device)? In these cases, granularity should be decreased to have enough slots for the sensor devices.

\section{Related Works}

WSN are related to several different research domains such as pervasive environment, and smart building systems. In this paper, we give a brief summary of the following research areas that are concerned by our study: energy issues in WSN, sensor network query processing (SNQP), sensor-based Smart Building Management Systems (SBMS).

Energy Issue in WSN. Wireless devices bring important constraints such as limited battery lifetime. [12] classifies the existing studies on energy consumption of wireless sensor devices into 3 subgroups: duty-cycle, data-driven and mobility-based approaches. Our sensor configuration based subscription management approach benefits from scheduled rendezvous of duty-cycling and adaptive sampling/transmission of data-driven approaches.

Sensor Network Query Processing. The main functionality of Sensor Network Query Processors (SNQP) is to handle continuous queries and sensor data streams $[13]$. [14, 15] propose an adaptive in-network aggregation operator ADAGA for query processing on sensor devices in order to filter and reduce the volume of sensor data. The main functionality of ADAGA is to regulate/adjust sensor activities based on energy levels and memory usage of sensor devices. As we propose in our own study, ADAGA is also capable of processing query parameters but the study does not tackle multi-application context and multi-modality of wireless devices as proposed in our own work.

We use the Service-oriented Continuous Query (SoCQ) Engine [1] to manage sensor data streams. SoCQ has a strong advantage from its rivals (especially SNEE [8]) as it supports multi-application contexts. However, it supports neither energy-awareness nor real-time sensor configuration. Our approach provides these features and improvement to the SoCQ Engine.

Among available technologies for SNQP, [8] is the closest study to our approach. This study deal with the conflict between the quality of service and sensor acquisition/transmission settings. Authors propose an optimization of application requests and the generation of a query execution plan. Generated 
execution plan provides a global view of the network and a schedule that shows when to execute a sensor action (sleep, listen, receive/send a packet). In terms of sensor scheduling and parametrization of a query, this approach is highly close to ours. Still, query execution plan covers a single query with a single expectation.

Sensor-based Building Systems. Some other studies focus on the design and data management aspects as well. Most of those approaches are proposed for a specific application and adopt a static configuration for sensor devices. Sensor-actuator based environment management systems are also studied in this area. [16] mentions that sensor-actuator interactions has a significant effect on efficient building monitoring. Moreover, the study points out that estimation of our daily activities has a crucial role on managing heterogeneous sensor devices in the environment and provides energy saving and longer lifespans. [5] indicates that clustering and energy efficiency have been considered in wide area of WSN applications and propose a mechanism in which clusters are dynamically built up by neighbor nodes, to save energy and prolong the network lifetime.

[17, 18] are the closest studies to ours. [17] presents intelligent building architecture based on a self-adapting intelligent gateway. [18] presents selfadapting algorithms for context-aware systems. These studies propose dynamic system management while processing user preferences. However, these studies are bounded by predefined building applications and the relation between application requirements and sensor configuration is not established. Besides, these approaches do not benefit from the potential reconfiguration of acquisition and transmission frequencies: sensor configuration stays static during the system lifetime. Moreover, energy consumption within the WSN is not considered as a major issue.

[19] is also close to our approach. Author implicates event-triggered dynamic sensor configuration. The study proposes a ZigBee-based intelligent self-adjusting sensor platform (ZiSAS) that can autonomously reconfigure middleware, network topology, sensor density, and sensing rate based on the environmental situation. However, unlike our proposition, user preferences, application requirements, query mechanism or data stream processing are not handled.

Overview of most known existing studies on Smart Building applications is summarized in Table 3. For the categorization of these studies, we benefit from the key functionalities of a Smart Building Management System 
Table 3: Overview of most known existing studies on Smart Building applications.

\begin{tabular}{|c|c|c|c|c|c|c|c|}
\hline Study & $\begin{array}{l}\text { Multi- } \\
\text { App }\end{array}$ & $\begin{array}{l}\text { Dynamic } \\
\text { User } \\
\text { Config. } \\
\end{array}$ & $\begin{array}{l}\text { Context- } \\
\text { Awareness }\end{array}$ & $\begin{array}{l}\text { Real- } \\
\text { Time } \\
\text { Sensor } \\
\text { Config. }\end{array}$ & $\begin{array}{l}\text { Energy- } \\
\text { Aware } \\
\text { Monitor- } \\
\text { ing }\end{array}$ & $\begin{array}{l}\text { Query } \\
\text { En- } \\
\text { gine }\end{array}$ & Experimentation \\
\hline Doukas et al. $[21]$ & - & - & $\sqrt{ }$ & - & - & - & Real Testbed \\
\hline Chen et al. $[22]$ & - & - & $\sqrt{ }$ & - & - & - & Simulation \\
\hline Byun et al. [19] & - & - & $\sqrt{ }$ & $\sqrt{ }$ & $\sqrt{ }$ & - & Real Testbed \\
\hline Xiang et al. [23] & - & - & $\sqrt{ }$ & - & - & $\sqrt{ }$ & Real Testbed \\
\hline Rutishauser et al. [24] & - & - & $\sqrt{ }$ & - & - & $\sqrt{ }$ & Real Testbed \\
\hline Servigne et al. $[25]$ & - & - & - & - & - & - & Real Testbed \\
\hline Mamidi et al. $[26]$ & - & - & $\sqrt{ }$ & - & - & - & Real Testbed \\
\hline Kailas et al. $[27]$ & - & - & $\sqrt{ }$ & - & - & - & Real Testbed \\
\hline Agarwal et al. [28] & - & - & $\sqrt{ }$ & - & - & - & Real Testbed \\
\hline Preisel et al. $[29]$ & - & - & - & - & $\sqrt{ }$ & - & Real Testbed \\
\hline Schor et al. $[30]$ & - & - & $\sqrt{ }$ & - & $\sqrt{ }$ & - & Real Testbed \\
\hline Li et al. $[31]$ & - & - & $\sqrt{ }$ & - & $\sqrt{ }$ & - & Real testbed \\
\hline 3SoSM & $\sqrt{ }$ & $\checkmark$ & $\sqrt{ }$ & $\sqrt{ }$ & $\sqrt{ }$ & $\sqrt{ }$ & $\begin{array}{l}\text { Real Testbed } \\
\text { and Simulation }\end{array}$ \\
\hline
\end{tabular}

(SBMS) [20]. The last line of the table is allocated for our approach 3SoSM. Our research motivation is that the existing approaches are mostly WSN-level techniques that do not tackle real-time dynamic interactions between application continuous queries and the physical environment, in a multi-application context where sensor devices are multi-modal and requirements are dynamic. Hence, this lack creates a gap between the computing environment and the physical environment, that can be both managed by pervasive applications.

To address this gap and propose a clear solution for multi-application monitoring systems, we design a sustainable multi-application monitoring system architecture for pervasive environments that collects application requirements for sensor data streams and optimizes them into sensor configurations. We propose energy-aware dynamic sensor device re-configuration (as a result of an optimization process) to lower energy consumption while fulfilling real-time application requirements. Compared to existing studies, our approach 3SoSM provides a multi-application mechanism and allows dynamicity for user configurations and network-aware sensor management while optimizing the sensor communication for enhancing energy consumption by real-time sensor configuration.

\section{Conclusion}

In this paper, we focus on one of the major challenges of pervasive monitoring systems like smart buildings: how to optimize/reduce the energy consumption of the monitoring architecture itself while managing sensor data streams. Existing studies do not tackle the energy consumption of the monitoring system and commonly adopt static configurations for sensor devices. 
Since application requirements are dynamic with the context, a dynamic sensor configuration can be a suitable option to solve this problem. We introduce a sustainable declarative monitoring architecture where we adopt the PEMS principles to separate application development and optimization of device interactions. We present our approach 3SoSM that is based on a WSN scheduling mechanism for sensor actions. We propose an optimization algorithm to find the optimal communication time slots. As an outcome, an optimized schedule generates SCO-PATTERNs to configure each device. We introduce our implementation 3SoSM Gateway that supports the optimization process for multiple parameterized subscriptions from dynamic application requirements. Our approach is validated by the experiments using the SoCQ Engine and a modified WSNet simulator. Moreover, our approach gives opportunities to use real testbed data and simulation data which is not so common in the pervasive environment research domain. Smart building applications are our target application, however, this approach can be applicable to other data-centric pervasive environments as well [2].

\section{Conflict of Interest}

The authors declare that there is no conflict of interests regarding the publication of this paper.

\section{References}

[1] Y. Gripay, F. Laforest, J.-M. Petit, Socq: A framework for pervasive environments, in: ISPAN'09, IEEE, 2009, pp. 154-159.

[2] S. Surdu, Y. Gripay, V.-M. Scuturici, J.-M. Petit, P-bench: benchmarking in datacentric pervasive application development, in: Transactions on Large-Scale Data-and Knowledge-Centered Systems XI, Springer, 2013, pp. 51-75.

[3] S. Servigne, Y. Gripay, O. Pinarer, J. Samuel, A. Ozgovde, J. Jay, Heterogeneous sensor data exploration and sustainable declarative monitoring architecture: Application to smart building, ISPRS Ann. Photogramm. Remote Sens. Spatial Inf. Sci IV-4/W1 (2016) 97-104.

[4] C. Perera, A. Zaslavsky, M. Compton, P. Christen, D. Georgakopoulos, Semanticdriven configuration of internet of things middleware, in: SKG'13, IEEE, 2013, pp. $66-73$. 
[5] P. G. V. Naranjo, M. Shojafar, H. Mostafaei, Z. Pooranian, E. Baccarelli, P-sep: a prolong stable election routing algorithm for energy-limited heterogeneous fogsupported wireless sensor networks, The Journal of Supercomputing 73 (2) (2017) $733-755$.

[6] O. Pinarer, Y. Gripay, S. Servigne, A. Ozgovde, Energy enhancement of multiapplication monitoring systems for smart buildings, in: International Conference on Advanced Information Systems Engineering, Springer, 2016, pp. 131-142.

[7] O. Pinarer, A. Ozgovde, Improving the energy efficiency of wearable computing units using on sensor fifo memory, International Journal of e-Education, e-Business, eManagement and e-Learning 5 (2) (2015) 105.

[8] I. Galpin, A. A. Fernandes, N. W. Paton, Qos-aware optimization of sensor network queries, The VLDB Journal 22 (4) (2013) 495-517.

[9] I. Cardei, M. Cardei, Energy-efficient connected-coverage in wireless sensor networks, International Journal of Sensor Networks 3 (3) (2008) 201-210.

[10] Wsnet / worldsens simulator, http://wsnet.gforge.inria.fr/.

[11] S. Cao, Z. Salcic, Z. Li, S. Wei, Y. Ding, Temperature-aware multi-application mapping on network-on-chip based many-core systems, Microprocessors and Microsystems 46 (2016) 149-160.

[12] G. Anastasi, M. Conti, M. Di Francesco, A. Passarella, Energy conservation in wireless sensor networks: A survey, Ad hoc networks 7 (3) (2009) 537-568.

[13] F. Jabeen, S. Nawaz, In-network wireless sensor network query processors: State of the art, challenges and future directions, Information Fusion 25 (2015) 1-15.

[14] A. Brayner, A. L. Coelho, K. Marinho, R. Holanda, W. Castro, On query processing in wireless sensor networks using classes of quality of queries, Information Fusion 15 (2014) 44-55.

[15] A. Brayner, A. Lopes, D. Meira, R. Vasconcelos, R. Menezes, An adaptive in-network aggregation operator for query processing in wsn, Journal of Systems and Software 81 (3) (2008) 328-342.

[16] F. Viani, F. Robol, A. Polo, P. Rocca, G. Oliveri, A. Massa, Wireless architectures for heterogeneous sensing in smart home applications: Concepts and real implementation, Proceedings of the IEEE 101 (11) (2013) 2381-2396.

[17] J. Byun, S. Park, Development of a self-adapting intelligent system for building energy saving and context-aware smart services, IEEE Transactions on Consumer Electronics 57 (1) (2011) 90-98. 
[18] T. Cioara, I. Anghel, I. Salomie, M. Dinsoreanu, G. Copil, D. Moldovan, A selfadapting algorithm for context aware systems, in: RoEduNet'10, IEEE, 2010, pp. $374-379$.

[19] J. Byun, B. Jeon, J. Noh, Y. Kim, S. Park, An intelligent self-adjusting sensor for smart home services based on zigbee communications, IEEE Transactions on Consumer Electronics 58 (3) (2012) 794-802.

[20] T. A. Nguyen, M. Aiello, Energy intelligent buildings based on user activity: A survey, Energy and buildings 56 (2013) 244-257.

[21] H. Doukas, K. D. Patlitzianas, K. Iatropoulos, J. Psarras, Intelligent building energy management system using rule sets, Journal of Building and Environment 42 (10) (2007) 3562-3569.

[22] H. Chen, P. Chou, S. Duri, H. Lei, J. Reason, The design and implementation of a smart building control system, in: ICEBE'09, Citeseer, 2009.

[23] S. Xiang, W. Wu, K.-L. Tan, Optimizing multiple data acquisition queries in sparse mobile sensor networks, in: MDM'12, IEEE, 2012, pp. 137-146.

[24] U. Rutishauser, J. Joller, R. Douglas, Control and learning of ambience by an intelligent building, IEEE Transactions on Systems, Man and Cybernetics, Part A: Systems and Humans 35 (1) (2005) 121-132.

[25] S. Servigne, Y. Gripay, J.-M. Deleuil, C. Nguyen, J. Jay, O. Cavadenti, R. Mebrouk, Data science approach for a cross-disciplinary understanding of urban phenomena: Application to energy efficiency of buildings, Procedia Engineering 115 (2015) 45-52.

[26] S. Mamidi, Y.-H. Chang, R. Maheswaran, Improving building energy efficiency with a network of sensing, learning and prediction agents, in: Proceedings of the 11th AAMAS, Vol. 1, IFAAMAS'12, 2012, pp. 45-52.

[27] A. Kailas, V. Cecchi, A. Mukherjee, A survey of communications and networking technologies for energy management in buildings and home automation, Journal of Computer Networks and Communications 2012.

[28] Y. Agarwal, B. Balaji, R. Gupta, J. Lyles, M. Wei, T. Weng, Occupancy-driven energy management for smart building automation, Journal of Power 50 (100) (2010) 150.

[29] M. Preisel, A. Diaz, W. Wimmer, Energy consumption of smart meters, Journal of Information and Communication Technologies (2013) 37.

[30] L. Schor, P. Sommer, R. Wattenhofer, Towards a zero-configuration wireless sensor network architecture for smart buildings, in: BuildSys'09, ACM, 2009, pp. 31-36. 
749

750

751

[31] C. Li, F. Meggers, M. Li, J. Sundaravaradan, F. Xue, H. Lim, A. Schlueter, Bubblesense: wireless sensor network based intelligent building monitoring, Proceedings of the ICT4S'13 (2013) 159-166. 